\title{
Energy bagging tree
}

\author{
Taoyun CaO, Xueqin Wang*, And Heping Zhang
}

This paper introduces Energy Bagging Tree (EBT) for multivariate nonparametric regression problems. The EBT makes use of a measure of dispersion constructed from a generalized Gini's mean difference as node impurity, and the tree split function therefore corresponds to the product of energy distance and descendants' proportions. As a nonparametric extension of the between-sample variation in the analysis of variance, this measure of dispersion serves well for EBT in understanding certain complex data. Extensive simulation studies indicate that EBT is highly competitive with existing regression tree methods. We also assess the performance of the EBT through a real data analysis on forest fires.

AMS 2000 subJect Classifications: Primary 62G08, $62 \mathrm{H} 20$; secondary $62 \mathrm{P} 12$.

KEYWORDS AND PHRASES: Multivariate nonparametric regression, Energy bagging tree, Energy distance, Generalized Gini's mean difference.

\section{INTRODUCTION}

Multivariate data, where the focus of multivariate refers to data with more than one response variable, have emerged in many scientific areas such as ecology, biometrics, econometrics, and medicine. It is often important to detect the relationships between multivariate response variables and explanatory variables. For instance, forest fires are a severe environmental issue that endangers human lives. It is important to timely assess the forest fire weather related factors and spatial location including rain, wind, temperature, relative humidity, $\mathrm{x}$-axis spatial coordinate, $\mathrm{y}$-axis spatial coordinate and examine the relationships with fuel moisture codes containing Fine Fuel Moisture Code, Duff Moisture Code, and Drought Code.

Multivariate regression trees (MRT), as a nonparametric data analysis tool, can explore the relationships between multivariate response variables and explanatory variables by building a tree-like model without assuming a specified relationship or a distribution for the response variables. This nonparametric regression method, as described by Death [6], is flexible for analyzing complex data, involving imbalance between covariates and nonlinear relationships between variables as well as high-order interactions, and its results are intuitive for interpretation. Consequently, it has become a

\footnotetext{
*Corresponding author.
}

practical and useful regression tool as a complement to the parametric regression models [6-12].

A number of impurity measures for MRT have been proposed in the literature comprising multivariate sums of squared deviations about the multivariate sample mean, the sums of squared pairwise dissimilarities, and the Manhattan distance [6]. The Mahalanobis distance is used as node impurity in [7]. Special cases and applications of MRT have been considered and presented by various authors [8-12].

However, as Zhang and Wang [13] pointed out, tree-based methods have two major limitations: tree structure can be unstable even with minor data perturbations, resulting in potentially unreliable prediction performance; and with a large number of variables and/or observations, one tree is either too complex or unlikely to summarize the essential information in the data. Bootstrapping and aggregating (Bagging) $[1,22]$ offered one option to alleviate these problems.

Bagging is proposed to improve the stability and accuracy of tree-based method used in nonparametric regression. It belongs to a broader class of ensemble methods through voting or model averaging [2-5]. So far, the bagging method has been developed primarily for a univariate response variable, however. The goal of this article is to extend bagging to the case with multivariate responses.

In this paper, we propose a novel bagging approach called Energy Bagging Tree (EBT) to nonparametric regression. EBT extends bagging by utilizing a measure of dispersion based on the generalized Gini's mean difference as the node impurity. This measure possesses useful properties for node splitting [14]. The split function turns out to be the product of energy distance with the descendants' proportions. And EBT reduces to bagging when there is only one response variable.

The energy distance was earlier introduced by Rizzo and Székely [15] as a nonparametric measure of the difference between two random variables. It can be used to compare two sets of multivariate data with arbitrary but same dimension, and hence can be used to test the heterogeneity (or homogeneity) of complex data.

The remainder of the paper is organized as follows: Section 2 introduces EBT, including impurity and split functions and generalization error. We reassure in Section 3 that bagging is a special case of EBT for a univariate response variable. Simulation studies are presented in Section 4, followed by a real data analysis. In our numerical examples, EBT is compared with other impurity functions for multivariate responses. We conclude with a few remarks in Section 5 . 


\section{ENERGY BAGGING TREE}

\subsection{Energy distance}

Given two independent $p$-dimensional random variables $X$ and $Y$ with $\mathrm{E}\|X\|^{\alpha}<\infty, \mathrm{E}\|Y\|^{\alpha}<\infty$ for $\alpha \in(0,2)$, where $\|\cdot\|$ denotes the Euclidean norm. The energy distance (with power $\alpha$ ) between $X$ and $Y$ is defined as

$$
\varepsilon_{\alpha}(X, Y)=2 E\|X-Y\|^{\alpha}-E\left\|X-X^{\prime}\right\|^{\alpha}-E\left\|Y-Y^{\prime}\right\|^{\alpha},
$$

where $X^{\prime}$ and $Y^{\prime}$ are the independent and identical random realizations of $X$ and $Y$, respectively [15].

Specially, if $X$ is uniformly distributed in the set $A=\left(a_{1}, \ldots, a_{n}\right)$ and $Y$ is uniformly distributed in $B=$ $\left(b_{1}, \ldots, b_{m}\right)$, let

$$
g_{\alpha}(A, B)=\frac{1}{n m} \sum_{i=1}^{n} \sum_{j=1}^{m}\left\|a_{i}-b_{j}\right\|^{\alpha},
$$

be a generalized Gini's mean difference. Then, the energy distance of $X$ and $Y$ can be written as follows,

$$
\varepsilon_{\alpha}(A, B)=2 g_{\alpha}(A, B)-g_{\alpha}(A, A)-g_{\alpha}(B, B) .
$$

Note that $\varepsilon_{\alpha}(A, B)=0$ if and only if $A=B$, that is two sets $\mathrm{A}$ and $\mathrm{B}$ are exactly the same. $\varepsilon_{\alpha}(A, B)$ is an empirical energy distance between $X$ and $Y$ if $A$ and $B$ are the samples of $X$ and $Y$, respectively.

\subsection{Node impurity and split functions}

Suppose there is a set of observations $A(\tau)=\left\{Y_{j}, j \in\right.$ $N(\tau)\}$ in node $\tau$, and let $N(\tau)$ be the size of $A(\tau)$. We define the node impurity at $\tau$ as the generalized Gini's mean difference in equation (1),

$$
i_{\alpha}(\tau)=g_{\alpha}(A(\tau), A(\tau)) .
$$

With a possible split $s$ at $\tau$, by the same token, $A\left(\tau_{L}\right)$ and $A\left(\tau_{R}\right)$ respectively denote the sets of observations in the left daughter node $\tau_{L}$ of node $\tau$, and right daughter node $\tau_{R}$ of node $\tau, N\left(\tau_{L}\right)$ and $N\left(\tau_{R}\right)$ denote their sizes and $p\left(\tau_{L}\right)$ and $p\left(\tau_{R}\right)$ denote their proportion in $A(\tau)$. Namely,

$$
\begin{aligned}
& p\left(\tau_{L}\right)=\frac{N\left(\tau_{L}\right)}{N(\tau)}, \\
& p\left(\tau_{R}\right)=\frac{N\left(\tau_{R}\right)}{N(\tau)} .
\end{aligned}
$$

$i_{\alpha}\left(\tau_{L}\right)$ and $i_{\alpha}\left(\tau_{R}\right)$ respectively denote the impurity function at two daughter nodes,

$$
\begin{aligned}
& i_{\alpha}\left(\tau_{L}\right)=g_{\alpha}\left(A\left(\tau_{L}\right), A\left(\tau_{L}\right)\right), \\
& i_{\alpha}\left(\tau_{R}\right)=g_{\alpha}\left(A\left(\tau_{R}\right), A\left(\tau_{R}\right)\right) .
\end{aligned}
$$

The split function is defined as

$$
\phi_{\alpha}(s, \tau)=i_{\alpha}(\tau)-p\left(\tau_{L}\right) i_{\alpha}\left(\tau_{L}\right)-p\left(\tau_{R}\right) i_{\alpha}\left(\tau_{R}\right) .
$$

172 T. Cao, X. Wang, and H. Zhang
It should be noted that

$$
i_{\alpha}(\tau)=g_{\alpha}(A(\tau), A(\tau))=\frac{1}{N(\tau)^{2}} \sum_{i=1}^{N(\tau)} \sum_{j=1}^{N(\tau)}\left\|Y_{i}-Y_{j}\right\|^{\alpha} .
$$

Thus

$$
\begin{aligned}
& \phi_{\alpha}(s, \tau)=\frac{1}{N(\tau)^{2}} \sum_{i=1}^{N(\tau)} \sum_{j=1}^{N(\tau)}\left\|Y_{i}-Y_{j}\right\|^{\alpha} \\
& -\frac{N\left(\tau_{L}\right)}{N(\tau)} \frac{1}{N\left(\tau_{L}\right)^{2}} \sum_{u=1}^{N\left(\tau_{L}\right)} \sum_{v=1}^{N\left(\tau_{L}\right)}\left\|Y_{u}-Y_{v}\right\|^{\alpha} \\
& -\frac{N\left(\tau_{R}\right)}{N(\tau)} \frac{1}{N\left(\tau_{R}\right)^{2}} \sum_{g=1}^{N\left(\tau_{R}\right)} \sum_{h=1}^{N\left(\tau_{R}\right)}\left\|Y_{g}-Y_{h}\right\|^{\alpha},
\end{aligned}
$$

among

$$
\begin{aligned}
& \sum_{i=1}^{N(\tau)} \sum_{j=1}^{N(\tau)}\left\|Y_{i}-Y_{j}\right\|^{\alpha}=\sum_{u=1}^{N\left(\tau_{L}\right)} \sum_{v=1}^{N\left(\tau_{L}\right)}\left\|Y_{u}-Y_{v}\right\|^{\alpha} \\
& +\sum_{g=1}^{N\left(\tau_{R}\right)} \sum_{h=1}^{N\left(\tau_{R}\right)}\left\|Y_{g}-Y_{h}\right\|^{\alpha}+2 \sum_{l=1}^{N\left(\tau_{L}\right)} \sum_{r=1}^{N\left(\tau_{R}\right)}\left\|Y_{l}-Y_{r}\right\|^{\alpha} .
\end{aligned}
$$

So we have obtained the following equation,

$$
\phi_{\alpha}(s, \tau)=p\left(\tau_{L}\right) p\left(\tau_{R}\right) \varepsilon_{\alpha}\left(A\left(\tau_{L}\right), A\left(\tau_{R}\right)\right) .
$$

The equation (4) connects the split function in equation (3) with the energy distance defined in equation (2). By the properties of the energy distance deduced in [15], $\phi_{\alpha}(s, \tau)$ is a measure of between-node dispersion. If $\alpha$ takes the value of 2 and the response variable is univariate, $\phi_{2}(s, \tau)$ is proportional to the between-sample variation (sum of squared deviations from the mean) in the analysis of variance. However, if $\alpha=1, \phi_{1}(s, \tau)$ differs from the between-sample dispersion induced by the sum of absolute deviations in [6] even for a univariate variable. Therefore, our proposed split function is not an extension for the measure of dispersion induced by $L_{\alpha}$-norm when $0<\alpha<2$.

We should note that the split function is closely related to the energy distance, maximizing the split function is not equivalent to maximizing the energy distance, because the sizes of daughter nodes are not fixed. In fact, the splitting optimization tends to balance between the sizes of the daughter nodes and the energy distance.

Once the node impurity function and splitting criterion are defined, we can follow the same algorithm in [1] to grow trees.

\subsection{Generalization error}

After having the node impurity function and splitting criterion, it is important to assess how closely a result of predicted (function of the covariables or inputs) fits the data 
(the outputs). This is now common practice in supervised learning problems, generalization error has been regard as an index for measuring. In the following we review it.

Given a sample $S=\left\{\left(X_{i}, Y_{i}\right), i=1, \cdots, n\right\}$, where $X_{i}=$ $\left(X_{i 1}, \ldots, X_{i u}\right)$ is the $i$ th observed value of $X=\left(X_{1}, \ldots, X_{u}\right)$ and $Y_{i}=\left(Y_{i 1}, \ldots, Y_{i v}\right)^{T}$ is the $i$ th observed value of $Y=$ $\left(Y_{1}, \ldots, Y_{v}\right)^{T}, i=1, \cdots, n$.

First, we draw $B$ bootstrap samples from $S$. Each bootstrap sample is obtained by sampling with replacement. Second, we grow the $b$ th multivariate regression tree $T^{* b}$ from the $b$ th bootstrap sample, $1 \leq b \leq B$. Let $\hat{\mu}^{* b}(\cdot)$ denote the $b$ th prediction function for a new observation. A bagging prediction of $Y$ with explanatory variables $X$ is obtained by averaging the predictions:

$$
\hat{\mu}_{b a g}(X)=\frac{1}{B} \sum_{b=1}^{B} \hat{\mu}^{* b}(X) .
$$

We use the out-of-bag (OOB) samples to assess the accuracy of the above bagging prediction. For $\left(X_{i}, Y_{i}\right) \in S$, let $O_{i}$ be the set of indices of $b$ 's such that the $b$ th bootstrap sample does not contain $\left(X_{i}, Y_{i}\right)$. The OOB prediction of $Y_{i}$ is

$$
\hat{\mu}_{O O B}\left(X_{i}\right)=\frac{1}{\left|O_{i}\right|} \sum_{b \in O_{i}} \hat{\mu}^{* b}\left(X_{i}\right)
$$

here $\left|O_{i}\right|$ denotes the size of $O_{i}$.

Finally, we use the following generalization error as a measure of prediction accuracy:

$$
P E_{b a g}=\frac{1}{n} \sum_{i=1}^{n}\left(Y_{i}-\hat{\mu}_{O O B}\left(X_{i}\right)\right)^{T}\left(Y_{i}-\hat{\mu}_{O O B}\left(X_{i}\right)\right) .
$$

\section{UNIVARIATE REGRESSION BAGGING}

In this section, we relate EBT for $\alpha=2$ to bagging method [1] where the sum of squares about the mean (SSM) is used as the impurity function.

Specifically, in bagging, the node impurity $i(\tau)$ at node $\tau$ was defined as follows:

$$
i(\tau)_{s s m}=\sum_{i=1}^{N(\tau)}\left(Y_{i}-\bar{Y}(\tau)\right)^{2}
$$

where $\bar{Y}$ is the average of $Y_{i}$ 's within node $\tau, N(\tau)$ is the number of samples within node $\tau$. And its split function was chosen as follows:

$$
\phi(s, \tau)=i(\tau)_{s s m}-i\left(\tau_{L}\right)_{s s m}-i\left(\tau_{R}\right)_{s s m},
$$

where $s$ is an allowable split, $\tau_{L}$ and $\tau_{R}$ are the left and right daughter nodes of node $\tau$ resulting from split $s$, respectively.

When $\alpha=2$, the node impurity $i_{2}(\tau)$ for EBT is written as follows:

$$
i_{2}(\tau)=\frac{1}{N(\tau)^{2}} \sum_{i=1}^{N(\tau)} \sum_{j=1}^{N(\tau)}\left(Y_{i}-Y_{j}\right)^{2}
$$

where $Y_{i}$ and $Y_{j}$ are within node $\tau, N(\tau)$ is the number of samples within node $\tau$ as before. Equation (3) with $\alpha=2$ is its corresponding split function. Namely,

$$
\phi_{2}(s, \tau)=i_{2}(\tau)-p\left(\tau_{L}\right) i_{2}\left(\tau_{L}\right)-p\left(\tau_{R}\right) i_{2}\left(\tau_{R}\right) .
$$

It follows from equation (2.4) in [15] that

$$
i_{2}(\tau)=\frac{2}{N(\tau)} i(\tau)_{s s m}
$$

Thus

$$
\phi_{2}(s, \tau)=\frac{2}{N(\tau)} \phi(s, \tau)
$$

Considering that $N(\tau)$ is a constant when splitting node $\tau$, the impurity and split functions between $\operatorname{EBT}(\alpha=2)$ and bagging are in effect the same for univariate response variable.

\section{MULTIVARIATE REGRESSION BAGGING}

In this section, we report simulation experiments and a real data analysis to demonstrate the potential of EBT in tree construction for multivariate response variables. We compare its performance with that of existing MRT methods in the context of bagging: bagging(SAM), bagging(SSM) and bagging(SMD) in order to perform an unbiased comparison. The bagging(SAM) uses the sums of the Manhattan distance (the sums of absolute pairwise deviations) as the impurity functions [6], and bagging(SSM) uses the sums of squares about the mean as the impurity functions [6], and bagging(SMD) exploits the sums of Mahalanobis distance as the impurity function [7]. As presented in [6] and [7], equation

$$
\phi(s, \tau)=i(\tau)-i\left(\tau_{L}\right)-i\left(\tau_{R}\right),
$$

is the corresponding split function in bagging(SAM), bagging(SSM) and bagging(SMD).

Note that equations (9) and (10) still hold for multivariate responses, and that $\operatorname{EBT}(\alpha=2)$ and bagging(SSM) are equivalent. Therefore, we primarily compare the following four methods, $\operatorname{EBT}(\alpha=1)$, bagging(SAM), bagging(SSM) and bagging(SMD). Also notice that $\alpha=1$ is the simplest choice within interval $\alpha \in(0,2]$ for energy distance, which is why we consider $\operatorname{EBT}(\alpha=1)$.

\subsection{General settings}

To assess the performance, we consider the following three criteria similar to those in [11] and [18].

1. $25 \%\left(Q_{1}\right), 50 \%$ (median), $75 \%\left(Q_{3}\right)$ quantiles of generalization errors. The generalization errors have emerged as one of the most commonly used indices to evaluate the predictive power of ensemble methods.

2. Mean of tree complexity is measured by the number of terminal nodes.

3. Frequency of a variable being selected in a tree. 
Table 1. Distributions of predictor variables

$X=\left(X_{1}, X_{2}, X_{3}, X_{4}, X_{5}, X_{6}\right)$ used in example 1 and 2, where $G$ a denotes Gamma distribution with shape parameter $k=2$ and scale parameter $\theta=2$, three dependent structures

among the $X$ variables: independent, weakly dependent, strongly dependent

\begin{tabular}{lccc}
\hline \hline & Independent & Weakly dependent & Strongly dependent \\
\hline$X_{1}$ & $\mathrm{Ga}$ & $X_{4}+X_{5}$ & $X_{4}+0.1 \mathrm{Ga}$ \\
$X_{2}$ & $\mathrm{Ga}$ & $\mathrm{Ga}$ & $\mathrm{Ga}$ \\
$X_{3}$ & $\mathrm{Ga}$ & $\mathrm{Ga}$ & $\mathrm{Ga}$ \\
$X_{4}$ & $\mathrm{Ga}$ & $\mathrm{Ga}$ & $\mathrm{Ga}$ \\
$X_{5}$ & $\mathrm{Ga}$ & $\mathrm{Ga}$ & $\mathrm{Ga}$ \\
$X_{6}$ & $\mathrm{Ga}$ & $\mathrm{Ga}$ & $\mathrm{Ga}$ \\
\hline
\end{tabular}

We simulated bivariate response variable $Y=\left(Y_{1}, Y_{2}\right)^{\prime}$, and normalized the values of the response variables to have zero mean and unit variance. The sample size $n$ is fixed to be 100 , and $\epsilon=\left(\epsilon_{1}, \epsilon_{2}\right)^{\prime}$ is a random error vector generated from the bivariate Cauchy distribution, the scale matrix $\sum=\left(\begin{array}{ll}1 & \rho \\ \rho & 1\end{array}\right)$, where correlation $\rho$ is set to be $0,0.5$ and 0.8 to evaluate the impact of correlation of $Y_{1}$ and $Y_{2}$ on the method. All simulations are replicated 100 times.

\subsection{Simulation study}

Example 1. In this example, data are generated as follows: $X=\left(X_{1}, \ldots, X_{6}\right)$ are six predictor variables, $Y_{1}, Y_{2}$ are response variables, and the distributions of the $X$ variables are given in Table 1. We consider three dependent structures among the $\mathrm{X}$ variables: independent, weakly dependent, strongly dependent. And,

$$
Y=\left(\begin{array}{c}
Y_{1} \\
Y_{2}
\end{array}\right)=\left(\begin{array}{c}
f(X)+\epsilon_{1} \\
f(X)+\epsilon_{2}
\end{array}\right)
$$

where

(1.a): $f(X)=2 X_{1}+2 X_{2}+2 X_{3}$, or

(1.b): $f(X)=5 X_{1} X_{2} I\left(A_{1}\right)+\exp \left(-\sqrt{X_{1}^{2}+X_{2}^{2}}\right) I\left(A_{2}\right)+$ $\sin \left(10 \pi X_{1} X_{2}\right) I\left(A_{3}\right)+\left(X_{1}+X_{2}\right) I\left(A_{4}\right)$.

Here $I(\cdot)$ is the indicator function. $A_{1}=\left\{X_{1} \leq 4, X_{2} \leq 4\right\}$, $A_{2}=\left\{X_{1}>4, X_{2} \leq 4\right\}, A_{3}=\left\{X_{1} \leq 4, X_{2}>4\right\}$, and $A_{4}=\left\{X_{1}>4, X_{2}>4\right\}$.

It is worth noting that we included some noise variables in the $X$ that are irrelevant to the responses. Furthermore, the regression function is linear in model (1.a), and contains a piecewise function with interaction terms, exponential function, periodic and non-monotonous function terms in model (1.b).

Table 2 provides a summary from 100 simulation runs. We can see that $\operatorname{EBT}(\alpha=1)$ has advantage in balancing between $Q_{1}$, median, $Q_{3}$ quantiles of generalization errors and tree complexity. $\operatorname{EBT}(\alpha=1)$ owns the lowest degree of tree complexity with the loss of the prediction performance. Figure 1 displays the bar graphs of the frequencies when a
Table 2. The $25 \%\left(Q_{1}\right), 50 \%$ (median), $75 \%$ ( $Q_{3}$ ) quantiles of generalization errors and the mean complexity in bagging in 100 replications for model (1.a)

\begin{tabular}{lccccr}
\hline \hline \multicolumn{5}{c}{ Independent X } \\
\hline$\rho$ & method & $Q_{1}$ & median & $Q_{3}$ & Nodes \\
\hline 0 & EBT $(\alpha=1)$ & 1.120 & 1.588 & 2.074 & 10.7 \\
& bagging(SSM) & 1.146 & 1.580 & 2.092 & 10.9 \\
& bagging(SAM) & 1.176 & 1.557 & 1.981 & 18.1 \\
& bagging(SMD) & 1.919 & 1.990 & 2.045 & 10.2 \\
0.5 & EBT $(\alpha=1)$ & 1.105 & 1.651 & 2.003 & 10.8 \\
& bagging(SSM) & 1.101 & 1.653 & 2.042 & 11.0 \\
& bagging(SAM) & 1.127 & 1.609 & 1.980 & 18.5 \\
& bagging(SMD) & 1.930 & 2.018 & 2.074 & 9.9 \\
0.8 & EBT( $\alpha=1)$ & 1.051 & 1.694 & 2.126 & 10.1 \\
& bagging(SSM) & 1.084 & 1.739 & 2.156 & 10.3 \\
& bagging(SAM) & 1.073 & 1.652 & 2.058 & 17.7 \\
& bagging(SMD) & 1.899 & 2.010 & 2.068 & 8.9 \\
\hline
\end{tabular}

\begin{tabular}{lccccr}
\hline \multicolumn{5}{c}{ Weakly dependent X } \\
\hline$\rho$ & method & $Q_{1}$ & median & $Q_{3}$ & Nodes \\
\hline 0 & EBT( $\alpha=1)$ & 1.169 & 1.599 & 2.080 & 10.1 \\
& bagging(SSM) & 1.146 & 1.610 & 2.147 & 10.3 \\
& bagging(SAM) & 1.130 & 1.537 & 2.106 & 17.3 \\
& bagging(SMD) & 1.826 & 1.971 & 2.052 & 10.1 \\
0.5 & EBT( $\alpha=1)$ & 0.912 & 1.282 & 1.741 & 10.7 \\
& bagging(SSM) & 0.907 & 1.300 & 1.782 & 10.9 \\
& bagging(SAM) & 0.917 & 1.298 & 1.729 & 17.8 \\
& bagging(SMD) & 1.770 & 1.905 & 2.015 & 10.8 \\
0.8 & EBT( $\alpha=1)$ & 0.919 & 1.384 & 1.983 & 10.1 \\
& bagging(SSM) & 0.924 & 1.418 & 2.068 & 10.3 \\
& bagging(SAM) & 0.932 & 1.345 & 1.939 & 17.5 \\
& bagging(SMD) & 1.821 & 1.947 & 2.014 & 10.0 \\
\hline
\end{tabular}

\begin{tabular}{lccccr}
\hline \multicolumn{5}{c}{ Strongly dependent X } \\
\hline$\rho$ & method & $Q_{1}$ & median & $Q_{3}$ & Nodes \\
\hline 0 & EBT $(\alpha=1)$ & 1.223 & 1.581 & 1.997 & 10.5 \\
& bagging(SSM) & 1.256 & 1.530 & 1.999 & 10.7 \\
& bagging(SAM) & 1.168 & 1.505 & 1.859 & 17.8 \\
& bagging(SMD) & 1.901 & 1.999 & 2.044 & 9.8 \\
0.5 & EBT( $\alpha=1)$ & 1.139 & 1.728 & 2.201 & 10.2 \\
& bagging(SSM) & 1.173 & 1.717 & 2.138 & 10.3 \\
& bagging(SAM) & 1.147 & 1.704 & 2.045 & 17.2 \\
& bagging(SMD) & 1.939 & 2.003 & 2.033 & 9.4 \\
0.8 & EBT( $\alpha=1)$ & 1.122 & 1.652 & 2.174 & 10.0 \\
& bagging(SSM) & 1.151 & 1.679 & 2.310 & 10.2 \\
& bagging(SAM) & 1.183 & 1.645 & 2.089 & 17.7 \\
& bagging(SMD) & 1.894 & 2.009 & 2.052 & 9.1 \\
\hline & & & & &
\end{tabular}

variable is selected in a tree by the four methods in model (1.a). It suggests that when predictors $X$ are independent, $\operatorname{EBT}(\alpha=1)$ has higher probability of selecting true predictors $X_{1}, X_{2}$ and $X_{3}$, and the lowest probability of selecting noise predictors $X_{4}, X_{5}$ and $X_{6}$. This is still the case for the weakly dependent $X$ variables. With strongly dependent $X$ variables, $\operatorname{EBT}(\alpha=1)$ maintains the lowest probability of selecting noise predictors $X_{5}$ and $X_{6}$, and relatively high probability of selecting the true predictors, especially for $X_{2}$ and $X_{3}$, while bagging(SAM) has the lowest probability of 

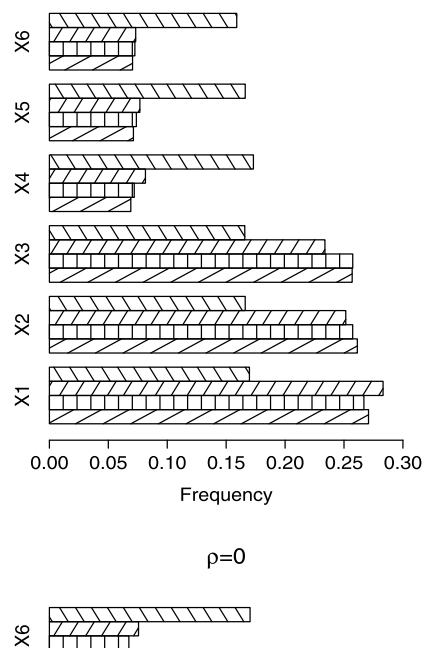

$\times$ ए人

• गोगो1110

2111

स गोगो

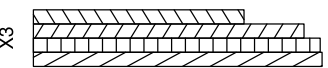

$\approx$

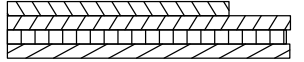

×

$\begin{array}{lllllll}0.00 & 0.05 & 0.10 & 0.15 & 0.20 & 0.25 & 0.30\end{array}$ Frequency

$\rho=0$

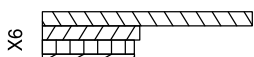

शीगोग11110

1111110

होगीय

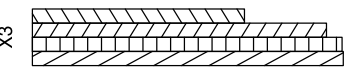

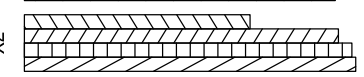

×

$\begin{array}{lllllll}0.00 & 0.05 & 0.10 & 0.15 & 0.20 & 0.25 & 0.30\end{array}$

Frequency

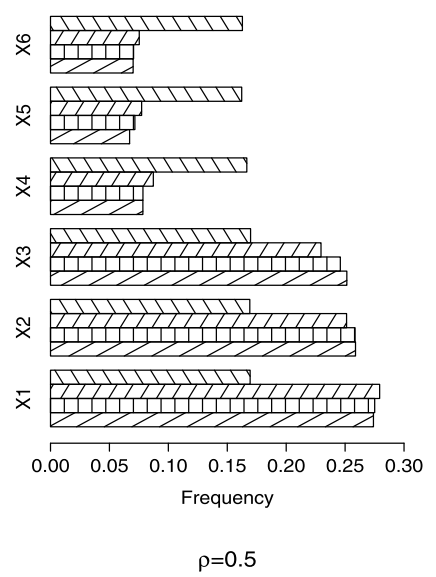

पोगोणा111

ए11

शीगो11110

2111

गोगोगा110

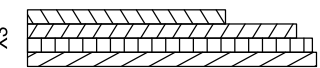

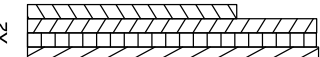

×

$\begin{array}{lllllll}0.00 & 0.05 & 0.10 & 0.15 & 0.20 & 0.25 & 0.30\end{array}$

Frequency

$\rho=0.5$

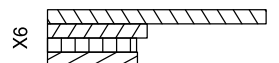

11111111118

×

* गोगी

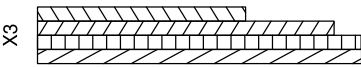

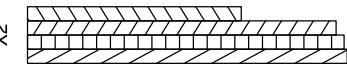

× शोगीणोगी

$\begin{array}{lllllll}0.00 & 0.05 & 0.10 & 0.15 & 0.20 & 0.25 & 0.30\end{array}$

Frequency

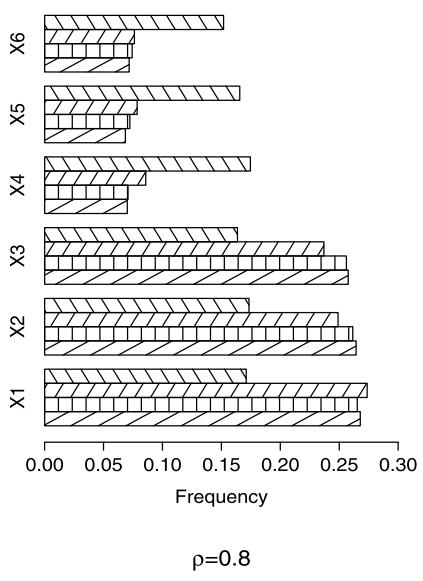

गोगीण1110

$\Rightarrow 2$

तोगा1110

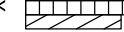

पोगोगा110

2111

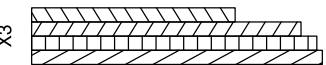

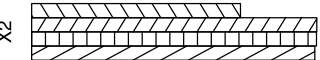

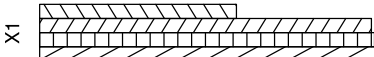

$\begin{array}{lllllll}0.00 & 0.05 & 0.10 & 0.15 & 0.20 & 0.25 & 0.30\end{array}$

Frequency

$\rho=0.8$

धोगोगा1111

$\times$ एणी

तोगोगा110

21111111

ष गोगि

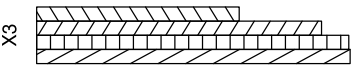

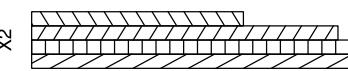

×

\begin{tabular}{lllllll}
\hline 0.00 & 0.05 & 0.10 & 0.15 & 0.20 & 0.25 & 0.30
\end{tabular}

Frequency

Figure 1. Frequencies of a variable selected by the four methods for model (1.a). Here in the rectangle oblique line (/) with $30^{\circ}$ : $\operatorname{EBT}(\alpha=1)$, vertical line $(\mid)$ : bagging $(S S M)$, oblique line $(/)$ with $60^{\circ}$ : bagging $(S A M)$, backslash $\left.\backslash\right)$ : bagging $(S M D)$.

The distributions of $X$ 's and three dependent structures among the $X$ variables are given in Table 1 . Horizontal axis shows frequencies of a variable selected, vertical axis shows six predictor variables. The first line denotes independent structure among the $X$ variables, weakly dependent structure and strongly dependent structure in the second line and third line, respectively. And each line shows three values of $\rho: 0,0.5$ and 0.8 .

selecting $X_{4}$ in the condition of strongly dependent between $X_{1}$ and $X_{4}$.

Table 3 reveals that $\operatorname{EBT}(\alpha=1)$ is superior to the other methods when variables $X$ are weakly dependent. It is clear that $\operatorname{EBT}(\alpha=1)$ owns the best prediction performance with the lowest degree of tree complexity when predictors $X$ are weakly dependent. In addition to this, $\operatorname{EBT}(\alpha=1)$ still has advantage in balancing between $Q_{1}$, median, $Q_{3}$ quantiles 
Table 3. The $25 \%\left(Q_{1}\right), 50 \%$ (median), $75 \%\left(Q_{3}\right)$ quantiles of generalization errors and the mean complexity in bagging in 100 replications for model (1.b)

\begin{tabular}{|c|c|c|c|c|c|}
\hline \multicolumn{6}{|c|}{ Independent X } \\
\hline$\rho$ & method & $Q_{1}$ & median & $Q_{3}$ & Nodes \\
\hline \multirow[t]{4}{*}{0} & $\operatorname{EBT}(\alpha=1)$ & 1.372 & 1.748 & 2.109 & 11.2 \\
\hline & bagging(SSM) & 1.465 & 1.818 & 2.200 & 11.3 \\
\hline & bagging(SAM) & 1.192 & 1.643 & 1.956 & 15.7 \\
\hline & bagging(SMD) & 2.022 & 2.050 & 2.096 & 8.5 \\
\hline \multirow[t]{4}{*}{0.5} & $\operatorname{EBT}(\alpha=1)$ & 1.115 & 1.466 & 2.016 & 11.4 \\
\hline & bagging(SSM) & 1.171 & 1.523 & 2.042 & 11.7 \\
\hline & bagging(SAM) & 1.071 & 1.332 & 1.816 & 15.9 \\
\hline & bagging(SMD) & 2.014 & 2.048 & 2.093 & 8.3 \\
\hline \multirow[t]{4}{*}{0.8} & $\operatorname{EBT}(\alpha=1)$ & 1.159 & 1.648 & 2.119 & 10.9 \\
\hline & bagging(SSM) & 1.255 & 1.679 & 2.180 & 11.2 \\
\hline & bagging(SAM) & 1.097 & 1.569 & 2.001 & 15.9 \\
\hline & bagging(SMD) & 2.014 & 2.036 & 2.098 & 7.5 \\
\hline \multicolumn{6}{|c|}{ Weakly dependent X } \\
\hline$\rho$ & method & $Q_{1}$ & median & $Q_{3}$ & Nodes \\
\hline \multirow[t]{4}{*}{0} & $\operatorname{EBT}(\alpha=1)$ & 1.278 & 1.646 & 2.077 & 8.4 \\
\hline & bagging(SSM) & 1.360 & 1.813 & 2.168 & 8.9 \\
\hline & bagging(SAM) & 1.359 & 1.769 & 2.070 & 12.7 \\
\hline & bagging(SMD) & 1.972 & 2.032 & 2.097 & 9.2 \\
\hline \multirow[t]{4}{*}{0.5} & $\operatorname{EBT}(\alpha=1)$ & 1.131 & 1.609 & 2.166 & 8.3 \\
\hline & bagging(SSM) & 1.356 & 1.739 & 2.228 & 8.8 \\
\hline & bagging(SAM) & 1.328 & 1.703 & 2.086 & 12.8 \\
\hline & bagging(SMD) & 1.957 & 2.027 & 2.086 & 8.8 \\
\hline \multirow[t]{4}{*}{0.8} & $\operatorname{EBT}(\alpha=1)$ & 1.100 & 1.506 & 2.055 & 8.3 \\
\hline & bagging(SSM) & 1.234 & 1.682 & 2.105 & 8.8 \\
\hline & bagging(SAM) & 1.195 & 1.687 & 2.057 & 13.1 \\
\hline & bagging(SMD) & 1.898 & 2.027 & 2.072 & 9.0 \\
\hline \multicolumn{6}{|c|}{ Strongly dependent X } \\
\hline$\rho$ & method & $Q_{1}$ & median & $Q_{3}$ & Nodes \\
\hline \multirow[t]{4}{*}{0} & $\operatorname{EBT}(\alpha=1)$ & 1.166 & 1.516 & 1.871 & 10.8 \\
\hline & bagging(SSM) & 1.212 & 1.594 & 1.964 & 11.1 \\
\hline & bagging(SAM) & 1.130 & 1.477 & 1.785 & 15.1 \\
\hline & bagging(SMD) & 2.011 & 2.053 & 2.106 & 8.4 \\
\hline \multirow[t]{4}{*}{0.5} & $\operatorname{EBT}(\alpha=1)$ & 0.993 & 1.425 & 1.799 & 10.8 \\
\hline & bagging(SSM) & 1.055 & 1.441 & 1.922 & 11.1 \\
\hline & bagging(SAM) & 0.930 & 1.319 & 1.787 & 15.1 \\
\hline & bagging(SMD) & 1.998 & 2.041 & 2.073 & 8.5 \\
\hline \multirow[t]{4}{*}{0.8} & $\operatorname{EBT}(\alpha=1)$ & 1.153 & 1.453 & 1.838 & 10.9 \\
\hline & bagging(SSM) & 1.184 & 1.500 & 1.851 & 11.2 \\
\hline & bagging(SAM) & 1.044 & 1.414 & 1.761 & 15.3 \\
\hline & bagging(SMD) & 2.003 & 2.041 & 2.082 & 8.0 \\
\hline
\end{tabular}

of generalization errors and tree complexity when predictors $X$ are independent or strongly dependent. Figure 2 displays the bar graphs of the frequencies when a variable is selected in a tree by the four methods in model (1.b). $\operatorname{EBT}(\alpha=1)$ has the highest probability of selecting true predictors $X_{1}$ and $X_{2}$ and the lowest probability of selecting noise predictors $X_{3}, X_{4}, X_{5}$, and $X_{6}$ in the case of $X$ are weakly dependent. However, bagging(SAM) has the lowest probability of selecting $X_{4}$ in the condition of strongly dependent between $X_{1}$ and $X_{4}$ as in model (1.a). $\operatorname{EBT}(\alpha=1)$ and bag-
ging(SSM) work very well in selecting the true variables $X_{1}$ and $X_{2}$, meantime excluding the noise variables for this relatively complex regression function. We should remind that bagging( $\mathrm{SSM})$ is equivalent to $\operatorname{EBT}(\alpha=2)$.

Example 2. In the first example, the bivariate $Y$ depends on a single regression function $f(X)$. In this example, distributions of predictor variables are used as before, we consider $Y$ depending on two regression functions $g(X)$ and $h(X)$. That is,

$$
Y=\left(\begin{array}{c}
Y_{1} \\
Y_{2}
\end{array}\right)=\left(\begin{array}{c}
g(X)+\epsilon_{1} \\
h(X)+\epsilon_{2}
\end{array}\right)
$$

Here,

(2.a): $g(X)=\sin \left(\pi X_{1} X_{2}\right)-X_{3}, h(X)=5 X_{1} X_{2}+2 X_{3}$.

We see that $g(X)$ and $h(X)$ are not linear and include interaction and periodic terms.

As before, the advantage of $\operatorname{EBT}(\alpha=1)$ in terms of balancing between $Q_{1}$, median, $Q_{3}$ quantiles of generalization errors and tree complexity are clear from Table 4 no matter what the dependent structures the variables $\mathrm{X}$ have. It should be noted that bagging(SAM) also has comparable performance with $\operatorname{EBT}(\alpha=1)$ and bagging(SSM) based on Figure 3. Moreover Figure 3 confirms the favorable performance of the three methods relative to bagging(SMD) for model (2.a).

Example 3. In this example, we have constructed data similar as the regression function involving five variables in [25]. We first simulate $X_{1}, X_{2}, \ldots, X_{10}$ i.i.d. from Uniform $(0,1)$. Then, the values of $Y$ is defined as follows:

$$
Y=\left(\begin{array}{c}
Y_{1} \\
Y_{2}
\end{array}\right)=\left(\begin{array}{c}
f(X)+\epsilon_{1} \\
10 f(X)+\epsilon_{2}
\end{array}\right)
$$

where

(3.a): $f(X)=10 \sin \left(\pi X_{1} X_{2}\right)+20\left(X_{3}-0.5\right)^{2}+10 X_{4}+5 X_{5}$.

Note that $X_{6}, X_{7}, \ldots, X_{10}$ are noise variables. And linear terms, interaction terms, periodic terms and high-order terms appear in model (3.a).

Table 5 compares the performance of the four methods in this example, and $\operatorname{EBT}(\alpha=1)$ is favorable to the other methods in terms of balancing between $Q_{1}$, median, $Q_{3}$ quantiles of generalization errors and tree complexity. It is clear from Figure 4 that $\operatorname{EBT}(\alpha=1)$ can distinguish the true variables $X_{1}, X_{2}, X_{3}, X_{4}, X_{5}$ from the noise variables $X_{6}, X_{7}, X_{8}, X_{9}, X_{10}$.

In summary, through three simulation examples and four models, Tables 2-5 and Figures 1-4 demonstrate that $\operatorname{EBT}(\alpha=1)$ performs better than the competing methods from the view of three criteria presented in Section 4.1. And EBT has an obvious advantage in distinguishing the true variables from the noise variables for all models in simulation study. It is of great importance for variable selection. 

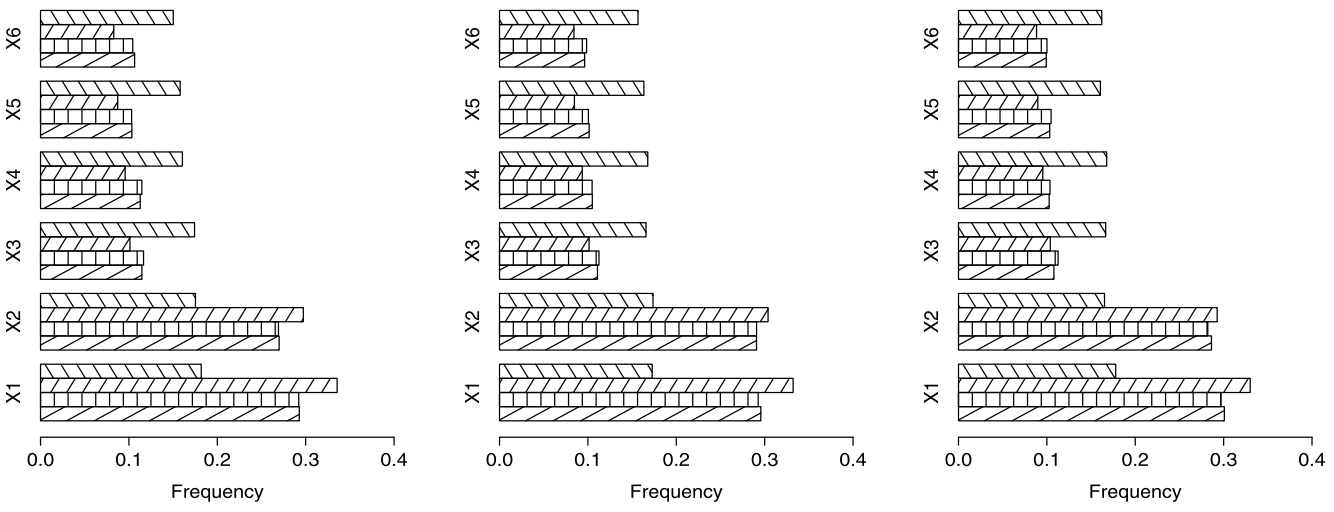

$\rho=0$

× गोगे

$\rho=0.5$

$\rho=0.8$

$\times$ होगी

च योगि

$\times$ गो111

×
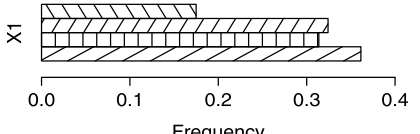

$\times$ शोगा11

एव

थ गोगाण

$\times$ सत

स गोगि

$\times$ योग

×

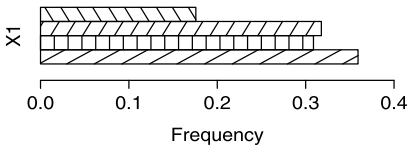

$\stackrel{8}{x}$

पोगा11

y

थ

¿211

艾

पोग

$\times$ ओग

$\approx$

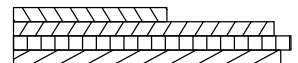

$\bar{x}$
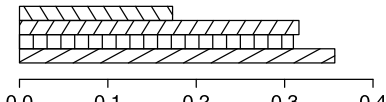

$\rho=0.5$

$\rho=0$

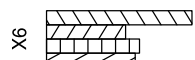

पोगमा11

2

स यौग

₹ योगा

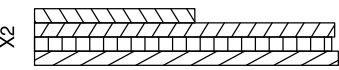

$\bar{x}$ पोगागायाग
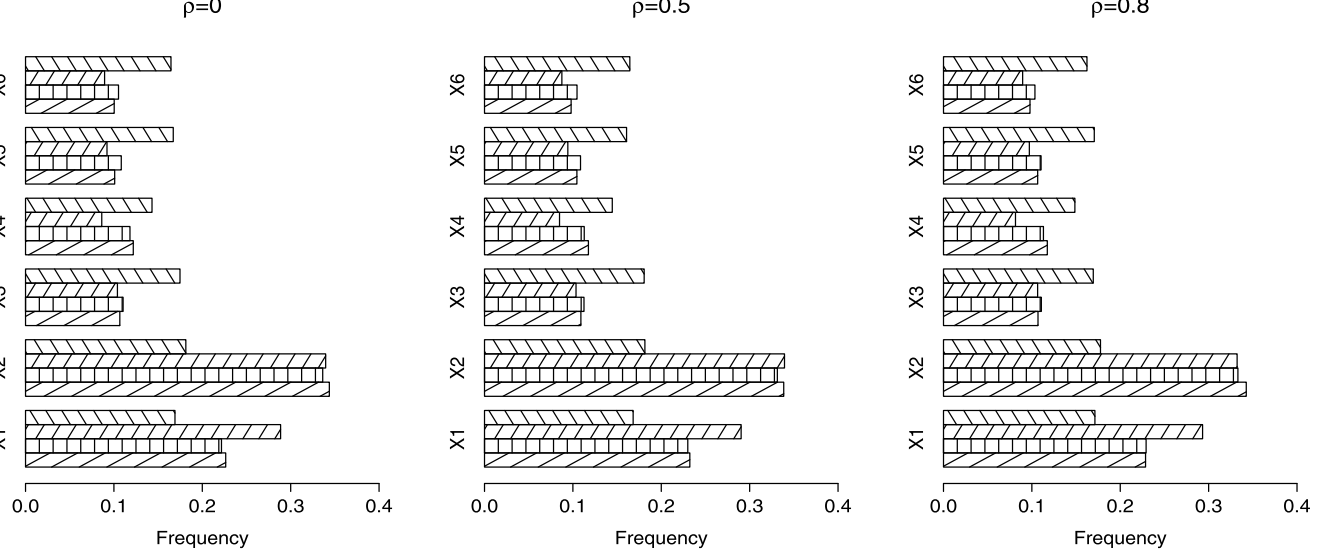

Figure 2. Frequencies of a variable selected by the four methods for model (1.b). Here in the rectangle oblique line (/) with $30^{\circ}$ : $\operatorname{EBT}(\alpha=1)$, vertical line $(\mid)$ : bagging $(S S M)$, oblique line $(/)$ with $60^{\circ}$ : bagging $(S A M)$, backslash $\left.\backslash\right)$ : bagging $(S M D)$.

The distributions of $X$ 's and three dependent structures among the $X$ variables are given in Table 1 . Horizontal axis shows frequencies of a variable selected, vertical axis shows six predictor variables. The first line denotes independent structure among the $X$ variables, weakly dependent structure and strongly dependent structure in the second line and third line, respectively. And each line shows three values of $\rho: 0,0.5$ and 0.8 .

\subsection{Case study}

Forest fires are a severe environmental issue while endangering human lives. A fast detection is critical to control and prevent such a disaster.
We use the Forest Fires dataset with a total of 517 entries, which is available from UCI Machine Learning Repository. Paulo Cortez and Anibal Morais [16] used the data to predict the burned area of forest fires via Data Mining approach. In 
Table 4. The $25 \%\left(Q_{1}\right), 50 \%$ (median), $75 \%\left(Q_{3}\right)$ quantiles of generalization errors and the mean complexity in bagging in 100 replications for model (2.a)

\begin{tabular}{|c|c|c|c|c|c|}
\hline \multicolumn{6}{|c|}{ Independent X } \\
\hline$\rho$ & method & $Q_{1}$ & median & $Q_{3}$ & Nodes \\
\hline \multirow[t]{4}{*}{0} & $\operatorname{EBT}(\alpha=1)$ & 1.318 & 1.415 & 1.584 & 10.5 \\
\hline & bagging(SSM) & 1.327 & 1.434 & 1.602 & 10.7 \\
\hline & bagging(SAM) & 1.260 & 1.374 & 1.494 & 17.9 \\
\hline & bagging(SMD) & 1.962 & 2.000 & 2.058 & 9.8 \\
\hline \multirow[t]{4}{*}{0.5} & $\operatorname{EBT}(\alpha=1)$ & 1.295 & 1.395 & 1.525 & 10.7 \\
\hline & bagging(SSM) & 1.319 & 1.427 & 1.552 & 10.9 \\
\hline & bagging(SAM) & 1.273 & 1.371 & 1.506 & 18.3 \\
\hline & bagging(SMD) & 1.959 & 2.016 & 2.069 & 10.1 \\
\hline \multirow[t]{5}{*}{0.8} & $\operatorname{EBT}(\alpha=1)$ & 1.300 & 1.404 & 1.579 & 10.3 \\
\hline & bagging(SSM) & 1.344 & 1.456 & 1.605 & 10.5 \\
\hline & bagging(SAM) & 1.253 & 1.407 & 1.571 & 17.6 \\
\hline & bagging(SMD) & 1.978 & 2.021 & 2.088 & 9.8 \\
\hline & \multicolumn{5}{|c|}{ Weakly dependent X } \\
\hline$\rho$ & method & $Q_{1}$ & median & $Q_{3}$ & Nodes \\
\hline \multirow[t]{4}{*}{0} & $\operatorname{EBT}(\alpha=1)$ & 1.249 & 1.374 & 1.481 & 10.7 \\
\hline & bagging(SSM) & 1.279 & 1.390 & 1.543 & 10.9 \\
\hline & bagging(SAM) & 1.223 & 1.321 & 1.421 & 18.2 \\
\hline & bagging(SMD) & 1.892 & 1.936 & 1.993 & 10.4 \\
\hline \multirow[t]{4}{*}{0.5} & $\operatorname{EBT}(\alpha=1)$ & 1.293 & 1.402 & 1.518 & 10.3 \\
\hline & bagging(SSM) & 1.320 & 1.416 & 1.542 & 10.5 \\
\hline & bagging(SAM) & 1.262 & 1.369 & 1.474 & 17.8 \\
\hline & bagging(SMD) & 1.886 & 1.947 & 2.017 & 10.1 \\
\hline \multirow[t]{4}{*}{0.8} & $\operatorname{EBT}(\alpha=1)$ & 1.260 & 1.369 & 1.474 & 10.8 \\
\hline & bagging(SSM) & 1.270 & 1.380 & 1.484 & 10.9 \\
\hline & bagging(SAM) & 1.204 & 1.329 & 1.427 & 18.3 \\
\hline & bagging(SMD) & 1.902 & 1.955 & 2.026 & 10.5 \\
\hline \multicolumn{6}{|c|}{ Strongly dependent X } \\
\hline$\rho$ & method & $Q_{1}$ & median & $Q_{3}$ & Nodes \\
\hline \multirow[t]{4}{*}{0} & $\operatorname{EBT}(\alpha=1)$ & 1.276 & 1.382 & 1.555 & 10.4 \\
\hline & bagging(SSM) & 1.313 & 1.436 & 1.598 & 10.6 \\
\hline & bagging(SAM) & 1.242 & 1.349 & 1.464 & 17.9 \\
\hline & bagging(SMD) & 1.936 & 2.000 & 2.059 & 9.8 \\
\hline \multirow[t]{4}{*}{0.5} & $\operatorname{EBT}(\alpha=1)$ & 1.240 & 1.369 & 1.524 & 10.4 \\
\hline & bagging(SSM) & 1.278 & 1.409 & 1.584 & 10.6 \\
\hline & bagging(SAM) & 1.208 & 1.327 & 1.469 & 17.8 \\
\hline & bagging(SMD) & 1.929 & 1.976 & 2.031 & 10.1 \\
\hline \multirow[t]{4}{*}{0.8} & $\operatorname{EBT}(\alpha=1)$ & 1.256 & 1.354 & 1.520 & 10.6 \\
\hline & bagging(SSM) & 1.286 & 1.412 & 1.564 & 10.8 \\
\hline & bagging(SAM) & 1.225 & 1.351 & 1.488 & 18.1 \\
\hline & bagging(SMD) & 1.911 & 1.969 & 2.030 & 10.2 \\
\hline
\end{tabular}

this work, we mainly explore the relationships between fuel moisture codes and six predictors, where fuel moisture codes contain Fine Fuel Moisture Code (FFMC), Duff Moisture Code (DMC), and Drought Code (DC). The six predictors are rain, wind, temperature, relative humidity, $\mathrm{x}$-axis spatial coordinate, y-axis spatial coordinate.

In our analysis, we made use of the resampling method. In every run, we chose 100 bootstrap samples, and replicated 100 times. Table 6 provides the three quantile results of the generalization errors. We can see that $\operatorname{EBT}(\alpha=1)$ 's gen-
Table 5. The $25 \%\left(Q_{1}\right), 50 \%$ (median), $75 \%$ ( $Q_{3}$ ) quantiles of generalization errors and the mean complexity in bagging in 100 replications for model (3.a)

\begin{tabular}{lccccc}
\hline \hline$\rho$ & method & $Q_{1}$ & median & $Q_{3}$ & Nodes \\
\hline 0 & EBT $(\alpha=1)$ & 1.265 & 1.422 & 1.684 & 12.3 \\
& bagging(SSM) & 1.259 & 1.459 & 1.701 & 12.5 \\
& bagging(SAM) & 1.270 & 1.421 & 1.610 & 19.7 \\
& bagging(SMD) & 1.793 & 1.885 & 1.954 & 11.9 \\
0.5 & EBT $(\alpha=1)$ & 1.229 & 1.438 & 1.677 & 12.3 \\
& bagging(SSM) & 1.221 & 1.449 & 1.745 & 12.5 \\
& bagging(SAM) & 1.227 & 1.414 & 1.612 & 19.8 \\
& bagging(SMD) & 1.803 & 1.889 & 1.972 & 11.7 \\
0.8 & EBT $(\alpha=1)$ & 1.219 & 1.416 & 1.696 & 12.2 \\
& bagging(SSM) & 1.228 & 1.442 & 1.781 & 12.5 \\
& bagging(SAM) & 1.241 & 1.377 & 1.628 & 19.7 \\
& bagging(SMD) & 1.791 & 1.908 & 1.970 & 11.5 \\
\hline
\end{tabular}

Table 6. The $25 \%\left(Q_{1}\right), 50 \%$ (median), $75 \%\left(Q_{3}\right)$ quantiles of generalization errors in Forest Fires data set in 100 replications

\begin{tabular}{cccc}
\hline \hline method & $Q_{1}$ & median & $Q_{3}$ \\
\hline $\operatorname{EBT}(\alpha=1)$ & 1.722 & 1.957 & 2.670 \\
bagging(SSM) & 1.693 & 1.982 & 2.791 \\
bagging(SAM) & 1.722 & 1.984 & 2.721 \\
bagging(SMD) & 2.258 & 2.539 & 3.206 \\
\hline
\end{tabular}

eralization errors are relatively lower than the other methods.

Figure 5 suggests that temperature and relative humidity are vital for FFMC, DMC and DC. Not surprisingly, the selected variables are important weather conditions for forest fires.

\section{DISCUSSION}

Bagging is known to be effective in exploring complex data structures. However, the conventional bagging method is generally used for univariate response only. In this paper, we have attempted to generalize bagging method for handling multivariate responses by using generalized Gini's mean difference as node impurity in constructing a tree during bagging. As such, the node split function corresponds to an adjusted energy distance. It should be noted that the split function of EBT is not an extension to the measure of dispersion induced by $L_{\alpha}$-norm, $0<\alpha<2$. The results from both simulation and real data analysis show that the proposed Energy Bagging Tree, $\operatorname{EBT}(\alpha=1)$ has its advantage than the existing MRT methods - bagging(SSM), bagging(SAM) and bagging(SMD) - when they are modified for bagging. We also noted that bagging(SSM) is equivalent to $\operatorname{EBT}(\alpha=2)$.

The advantage of bagging(SSM) lies in normal distribution for random error vector $\epsilon$. Bagging(SAM) has advantage for analysis of complex data structure, such as eco- 


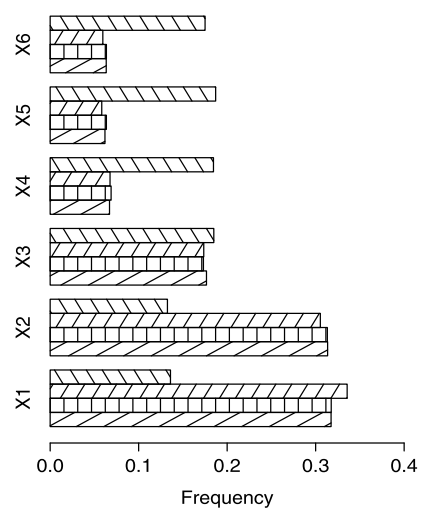

$\rho=0$
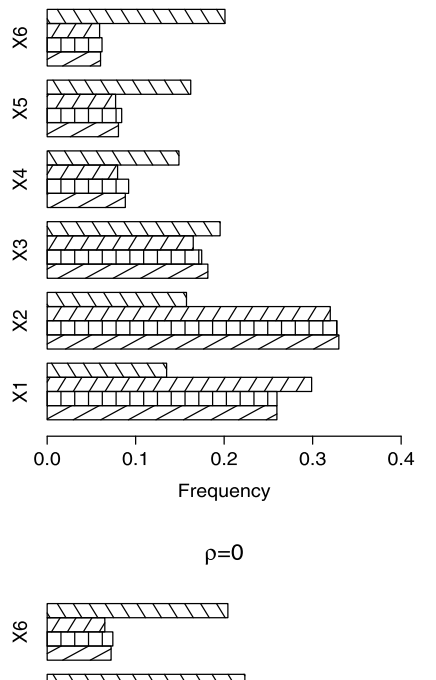

की

$\times$ \&

स पोग

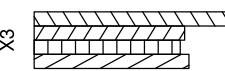

$\approx$ ओोगोगोणायाया

×

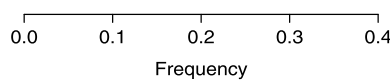

监

×

से गा111

$\times$ MIगमा

× होगोणीयायायु

×

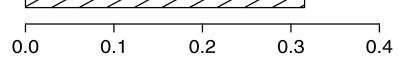

Frequency

$\rho=0.5$

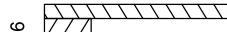

$\times$ 11

₹ गोगी

गो1110

* 210

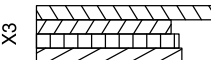

×

× शीगीय

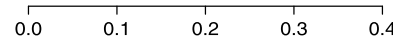

Frequency

$\rho=0.5$

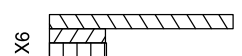

rz

$\times$ IोI111110

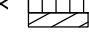

* गोगा

क गोगी।ीता

होगोोोग

Z Z Z Z

×

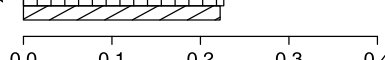

2 近

हो

स योगा11

यागमाल

₹

$\bar{x}$
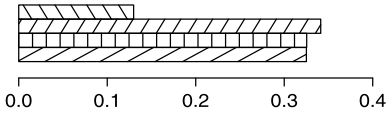

Frequency

$\rho=0.8$

6 गो1111110

$\times 20$

थ तोगा11

$z<$

स गोगे

₹ योगोणा

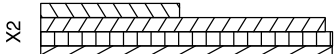

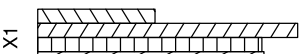

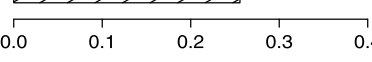

Frequency

$\rho=0.8$

$\times$ गोली

2

शोग111111

शी।

स गोगि

$\times$ ओोगोलो।

IIIIIIn

×

×

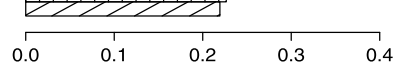

Figure 3. Frequencies of a variable selected by the four methods for model (2.a). Here in the rectangle oblique line (/) with $30^{\circ}$ : $\operatorname{EBT}(\alpha=1)$, vertical line (|): bagging $(S S M)$, oblique line (/) with $60^{\circ}$ : bagging(SAM), backslash $\left.\backslash\right)$ : bagging(SMD).

The distributions of $X$ 's and three dependent structures among the $X$ variables are given in Table 1 . Horizontal axis shows frequencies of a variable selected, vertical axis shows six predictor variables. The first line denotes independent structure among the $X$ variables, weakly dependent structure and strongly dependent structure in the second line and third line, respectively. And each line shows three values of $\rho: 0,0.5$ and 0.8 .

logical data with high-order and logarithm relationships between variables. And Bagging(SMD) has advantage for analysis of data with the simultaneous cooccurrence of several dependent variables, due to its impurity function is a variation of the approach of dealing with longitudinal data.

The main advantage of our proposed EBT is in two aspects: one is that it works for multivariate response variables 

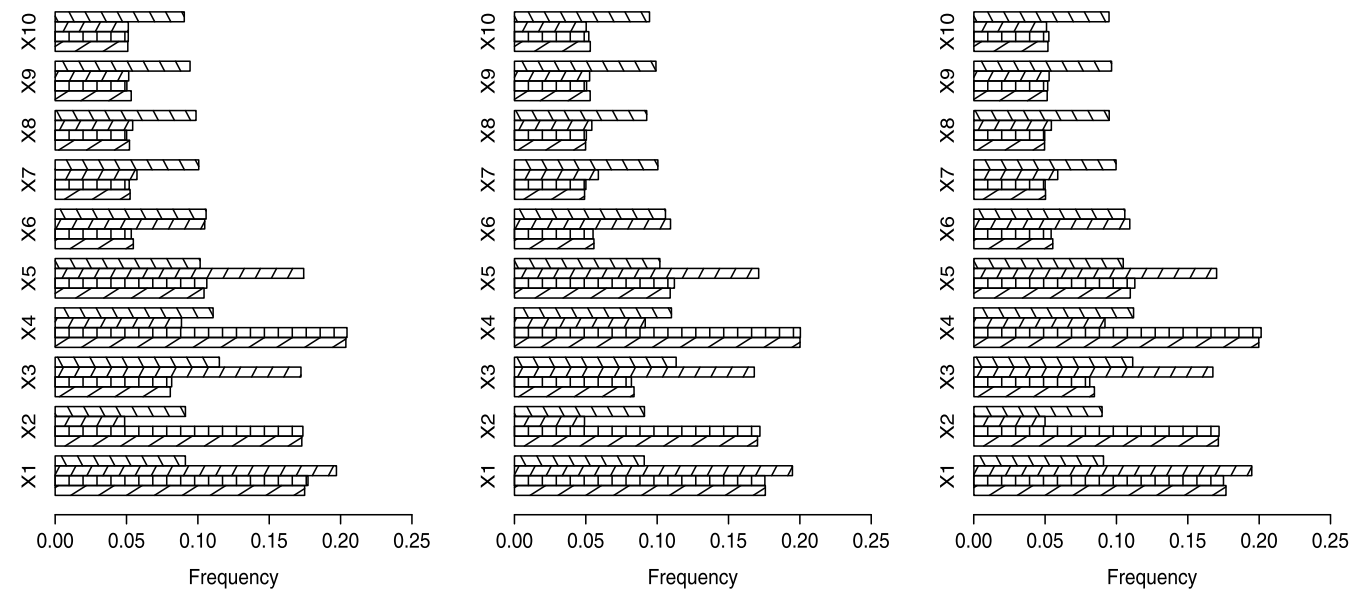

Figure 4. Frequencies of a variable selected by the four methods for model (3.a). Here in the rectangle oblique line (/) with $30^{\circ}$ : $\operatorname{EBT}(\alpha=1)$, vertical line ( $)$ : bagging(SSM), oblique line (/) with $60^{\circ}$ : bagging $(S A M)$, backslash $\left.\backslash\right)$ : bagging $(S M D)$.

The distributions of $X$ 's is from Uniform $(0,1)$. Horizontal axis shows frequencies of a variable selected, vertical axis shows ten predictor variables. And three values of $\rho: 0,0.5$ and 0.8 .

\section{Forest Fires}

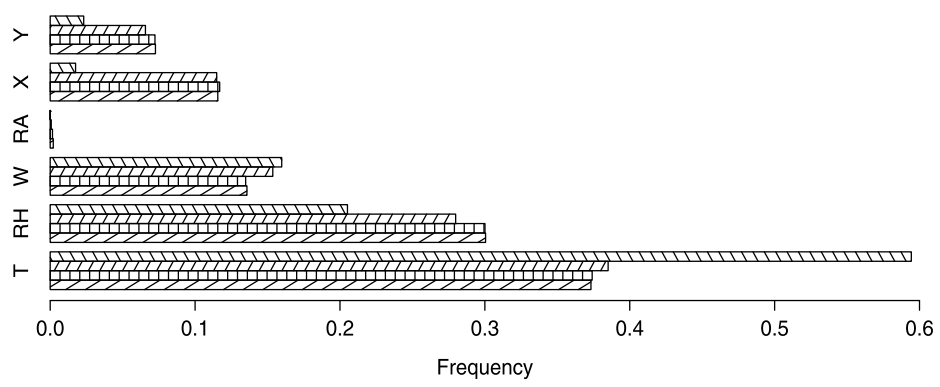

Figure 5. Frequencies of a variable selected by the four methods in the Forest Fires. Here in the rectangle oblique line (/) with $30^{\circ}$ : $\operatorname{EBT}(\alpha=1)$, vertical line (|): bagging(SSM), oblique line (/) with $60^{\circ}$ : bagging $(S A M)$, backslash $(\backslash)$ : bagging $(S M D)$. Horizontal axis shows frequencies of a variable selected, vertical axis shows six predictor variables. Here $T$ : temperature, RH: relative humidity, $W$ : wind, $R A$ : rain, $X$ : x-axis spatial coordinate, $Y$ : $y$-axis spatial coordinate.

and extends the scope of bagging tree. In the meantime, it covers bagging as a special case for univariate response variable and it covers multivariate sums of squared deviations about the multivariate sample mean [6] as a special case for multivariate response variables. The other is that it has the potential to be applied to variable selection because EBT has obvious advantage in distinguishing the true variables from the noise variables for four models in simulation studies.

Some issues deserve further study. For example, it may be interesting to explore whether we can choose $\alpha$ values to further improve the performance of EBT. We should note the limitation of our proposed EBT in that it deals with the quantitative response variables only. For multivariate discrete responses we need to further develop our method.

\section{ACKNOWLEDGEMENT}

Xueqin Wang's research is partially supported by NSFC for Excellent Young Scholar (11322108), NCET (12-0559), NSFC (11001280), RFDP (20110171110037). Heping Zhang's research is partially supported by grant R01DA016750-08 from the U.S. National Institute on Drug Abuse, a 1,000-plan scholarship from the Chinese Government, and the overseas and Hong Kong, Macau Young Scholars Collaborative Research Fund from the National Natural Science Foundation of China (11328103).

Received 21 January 2015

\section{REFERENCES}

[1] Breiman (1996). Bagging predictors. Machine Learning. 24123 140. 
[2] Hothorn, T. and Lausen, B. (2003). Bagging tree classifiers for laser scanning images: a data- and simulation-based strategy. Artificial Intelligence in Medicine. 27 65-79.

[3] Dietterich, T. G. (2000). An experimental comparison of three methods for constructing ensembles of decision trees: bagging, boosting, and randomization. Machine Learning. 40139 157.

[4] Hothorn, T. and Lausen, B. (2005). Bundling classifiers by bagging trees. Computational Statistics and Data Analysis. 491068 1078. MR2143058

[5] Prasad, A. M., Iverson, L. R., and Liaw, A. (2006). Newer classification and regression tree techniques: bagging and random forests for ecological prediction. Ecosystems. 9 181-199.

[6] Death, G. (2002). Multivariate regression trees: a new technique for modeling species-environment relationships. Ecology. $\mathbf{8 3}$ 1105-1117.

[7] Larsen, D. R. and Speckman, P. L. (2004). Multivariate regression trees for analysis of abundance data. Biometrics. $60543-549$. MR2067013

[8] Davidson, T. A., Sayer, C. D., Perrow, M., Bramm, M., and JePpesen, E. (2010). The simultaneous inference of zooplanktivorous fish and macrophyte density from sub-fossil cladoceran assemblages: a multivariate regression tree approach. Freshwater Biology. 55 546-564.

[9] Hamann, A., Gylander, T., and Chen, P.-Y. (2011). Developing seed zones and transfer guidelines with multivariate regression trees. Tree Genetics Genomes. 7 399-408.

[10] Questier, F., Put, R., Coomans, D., Walczak, B., and VanDER Heyden, Y. (2005). The use of CART and multivariate regression trees for supervised and unsupervised feature selection. Chemometrics and Intelligent Laboratory Systems. 76 4554.

[11] Hsiao, W.-C. and SHin, Y.-S. (2007). Splitting variable selection for multivariate regression trees. Statist. Probab. Lett. 77265 271. MR2339030

[12] Molinaro, A. M., Dudoit, S., and van der LaAn, M. J. (2004) Tree-based multivariate regression and density estimation with right-censored data. Journal of Multivariate Analysis. $90154-$ 177. MR2064940

[13] Zhang, H. and WANG, M. (2009). Search for the smallest random forest. Statistics and Its Interface. 2 381-396. MR2540095

[14] Yitzhaki, S. (2003). Gini's Mean difference: a superior measure of variability for non-normal distributions. International Journal of Statistics. lxi 285-316. MR2025523

[15] Rizzo, M. L. and SzÉKely, G. J. (2010). Disco analysis: a nonparametric extension of analysis of variance. The Annals of Ap plied Statistics. 4 1034-1055. MR2758432

[16] Cortez, P. and Morais, A. (2007). A data mining approach to predict forest fires using meteorological data. Home page: http://www.dsi.uminho.pt/pcortez.

[17] Izenman, A. J. (2008). Modern Multivariate Statistical Techniques. Springer, New York. MR2445017

[18] LOH, W.-Y. and ZHENG, W. (2013). Regression trees for longitudinal and multiresponse data. The Annals of Applied Statistics. 7 495-522. MR3086428

[19] Segal, M. R. (1992). Tree structured methods for longitudinal data. J. Amer. Statist. Assoc. 87 407-418.
[20] Zhang, H. (1998). Classification trees for multiple binary responses. J. Amer. Statist. Assoc. 93 180-193.

[21] Zhang, H. and Ye, Y. (2008). A tree-based method for modeling a multivariate ordinal response. Stat. Interface. 1 169-178. MR2425353

[22] Zhang, H. and Singer, B. H. (2010). Recursive Partitioning and Applications. Springer, New York. MR2674991

[23] Segal, M. and Xiao, Y. (2011). Multivariate random forests. Data Mining and Knowledge Discovery. 1 80-87.

[24] Breiman, L., Friedman, J. H., Olshen, R. A. and Stone, C. J. (1984). Classification and Regression Trees. Wadsworth, Belmont, CA.

[25] FRIEDMAN, J. H. (1991). Multivariate adaptive regression splines. The Annals of Statistics. 19 1-67. MR1091842

[26] Ahmad, A. and Brown, G. (2014). Random projection random discretization ensembles: ensembles of linear multivariate decision trees. IEEE Transactions on Knowledge and Data Engineering. 26 1225-1239.

Taoyun Cao

Southern China Research Center of Statistical Science

School of Mathematics and Computational Science

Sun Yat-Sen University

Guangzhou, 510275

China

E-mail address: caotaoyun@126.com

\section{Xueqin Wang}

Southern China Research Center of Statistical Science

School of Mathematics and Computational Science

Sun Yat-Sen University

Guangzhou, 510275

China

Zhongshan School of Medicine

Sun Yat-Sen University

Guangzhou, 510080

China

E-mail address: wangxq88@mail.sysu.edu.cn

Heping Zhang

Southern China Research Center of Statistical Science

School of Mathematics and Computational Science

Sun Yat-Sen University

Guangzhou, 510275

China

Department of Biostatistics

Yale University School of Public Health

New Haven, CT 06520-8034

USA

E-mail address: heping.zhang@yale.edu 